\title{
A VLA SURVEY OF MAGNETIC CATACLYSMIC VARIABLE STARS
}

\author{
Candace (iray ${ }^{1}$ and Paul A. Mason ${ }^{1}$
}

\section{Introduction}

Cataclysmic Variables (CVs) are close binaries containing a white dwarf primary and a Rochelobe filling red dwarf secondary. In magnetic CVs (MCVs) the white dwarfs have magnetic fields that are sufficiently strong $\left(10^{6}-10^{8}\right.$ Gauss $)$ to direct the accretion flow onto the surface of the primary. MCVs are divided into the lower field intermediate polars (IPs) and the higher field polars. Typically, IPs have accretion disks that are disrupted in the center and magnetically channelled flow onto the poles. Polars are diskless, an accretion stream flowing from the inner Lagrangian point impacts directly onto one or both magnetic poles. In addition, polars tend to have white dwar.s which rotate in synchronism with the binary orbit, while IPs have white dwarfs which rotate faster than the binary period. There are a few rare exceptions to this characterization, such as the slightly asynchronous polars and the stream-fed IPs.

\section{Previous Work}

Surveys of CVs suggest that non-magnetic CVs (outside of outburst) are not radio emitters (Cordova, Mason, and Hjellming 1983 and Fuerst, et al., 1986). However, some MCVs have been reported to be radio sources. Including three polars, three IPs; as well as the probable IP: FIRST J1023+0038.

The polars are AM Her, V834 Cen and ST LMi. AM Her exhibits quiescent non-thermal emission $(0.67 \mathrm{mJy})$ and a $100 \%$ circularly polarized flare (9.7 $\mathrm{mJy}$ ), both at $4.9 \mathrm{GHz}$ (Chanmugam and Dulk 1982). V834 Cen has strongly variable emission at $8.4 \mathrm{GHz}(2 \mathrm{mJy}$ ) with multiple flare events (up to 35 mJy) (Wright et al. 1988). There is marginal evidence that the emission is a function of orbital phase (Wright et al. 1988). And ST LMi was detected (2.0 $\pm 0.3 \mathrm{mJy}$ at $5 \mathrm{GHz}$ ) on 2 out of 3 occasions. This is a $6 \sigma$ detection, but Beasley et al. (1994) warn that confusion may have occurred.

The IPs are AE Aqr, DQ Her, BG CMi and the probable IP, J1023+0038. AE Aqr has variable non-thermal emission (1-12 $\mathrm{mJy}$ at $15 \mathrm{GHz}$ ) with multiple flare events (up to $35 \mathrm{mJy}$ ) (e.g. Bastian, Dulk, and Chanmugam 1988). It is a strong variable source across the spectrum, including $\gamma$-rays

\footnotetext{
${ }^{1}$ The University of Texas at El Paso, Department of Physics, El Paso, TX, 79968, USA (clgray@utep.edu).
}

at $\mathrm{TeV}$ energies (Mrintjes et al. 1992, de Jager et al. 1994). DQ Her was clevected (1-4 mJy at $5 \mathrm{GHz}$ ) on 3 of 4 oc(cisions, with a flaring erent (Pavelin et al. 1994). Strong upper limits on important MCVs have been obcained (Mason, Fisher, and Chanmugam 1996 and Beasley et al. 1994) An exciting result is $\mathrm{J} 1023+0038$, a probable $\mathrm{MC} \mathrm{C}$ with highly variable emission including a $6.6 \mathrm{~m}$.Jy flare at 1.4 GHz (Bond, White, Bucker, and O'Brien, 2002).

\section{The Survey}

We have begun a VLA survey of magnetic C'V in order to place strong constraints on models of radio emission by (1) completing the sample of all MCVs closer than $100 \mathrm{pc}$ and by (2) observing binaries with system properties similar to the quiescent and flaring radio MCV, AM Herculis. In addition, we shall attempt to confirm the detection of the first radio selected MICV, FIRST J1023+0038. Previous VLA surveys have shown that only a small fraction of MCVs are radio sources. However, these surveys have failed establish linkages between sw'stem properties such as magnetic field strength and the presence of radio emission. Such connections are vital in order to place constraints on radio emission mechanism models. Shortly after this meeting, data reduction began and the high field polar AR UNa was found to be a radio source.

\section{REFERENCES}

Bastian, T.S., Dulk, G., and Chanmugam, G. 1988. łpJ. 324,431

Beasley, A.J., Bastian, T.S, Ball, L., and Wu. K., 1994. AJ, 108, 2207

Bond, H.E., White, R.L., Becker, R.H.. and O'Brien. M.S. 2002, PASP, 11. 1359

Chanmugam, G., and Dulk, G.A. 19\$2. ApJ, 25.5. L107

Cordova, F.A., Mason, K.O., and Hjellming, R.M. 1983. PASP, 95, 96

De Jager, O.C., Meintjes, P.J., O'Donoghue, D.. and Robinson, E.L. 1994, MNRAS, 267. 577

Fuerst, E., Benz, A., Hirth, W.. Kiplinger, A., and Geffert, M. 1986, A\& A, 154. 377

Mason, P. A., Fisher, P. L., and Chanmugam G. 1996. A\& A, 310, 132

Meintjes et al. 1992, ApJ, 401, 325

Pavelin, P.E., et al. 1994. MNRAS. 269. 7T9

Wright, A.E., Cropper, M.S., Stewart, R.T. Nelsun. G.J., and Slee, O.B. 1988. MNR.LS. 231, 319 